
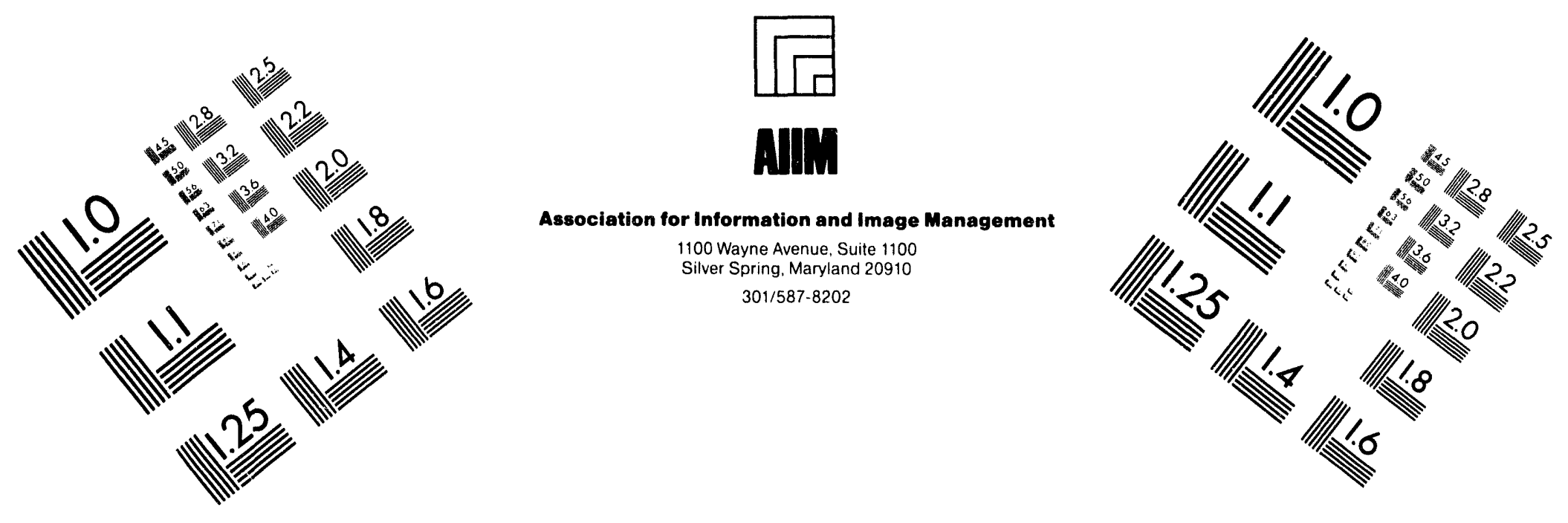

\title{
Centimeter
}

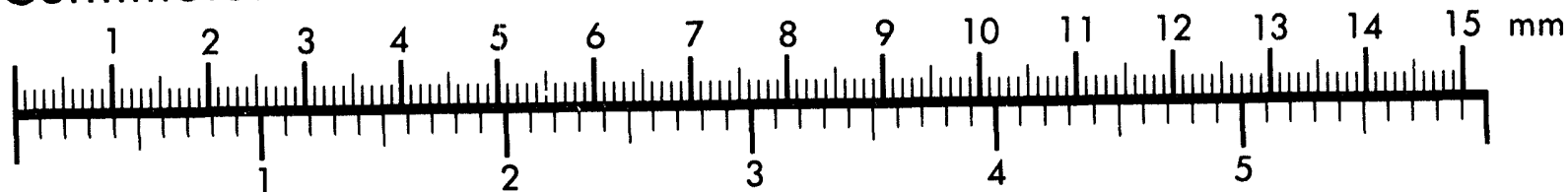
Inches
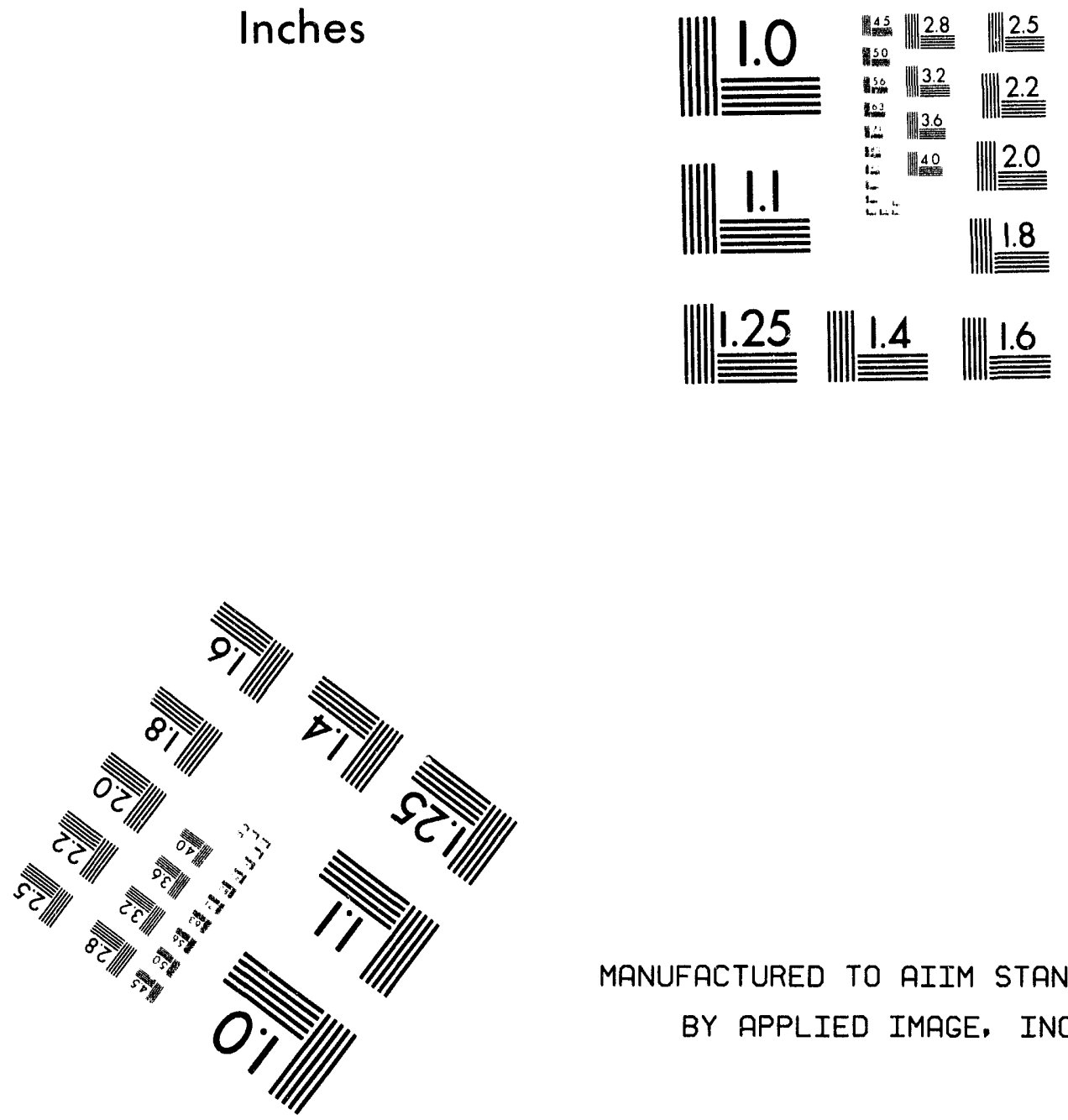

MANUFACTURED TO AIIM STANDARDS

BY APPLIED IMAGE, INC.

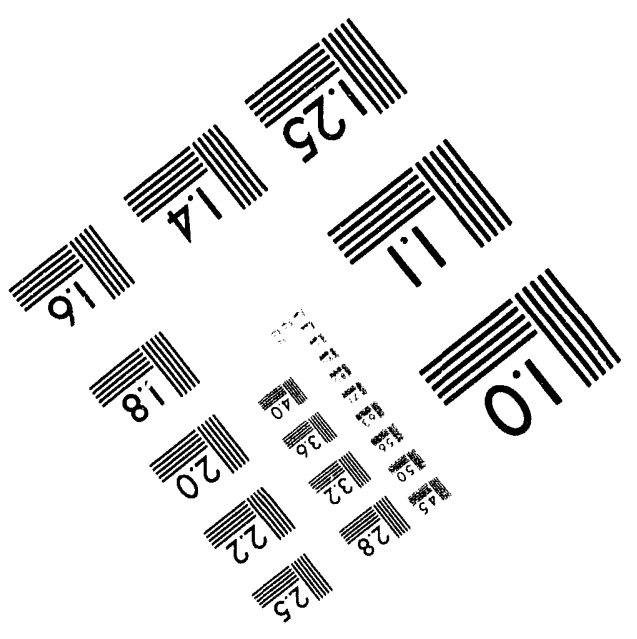



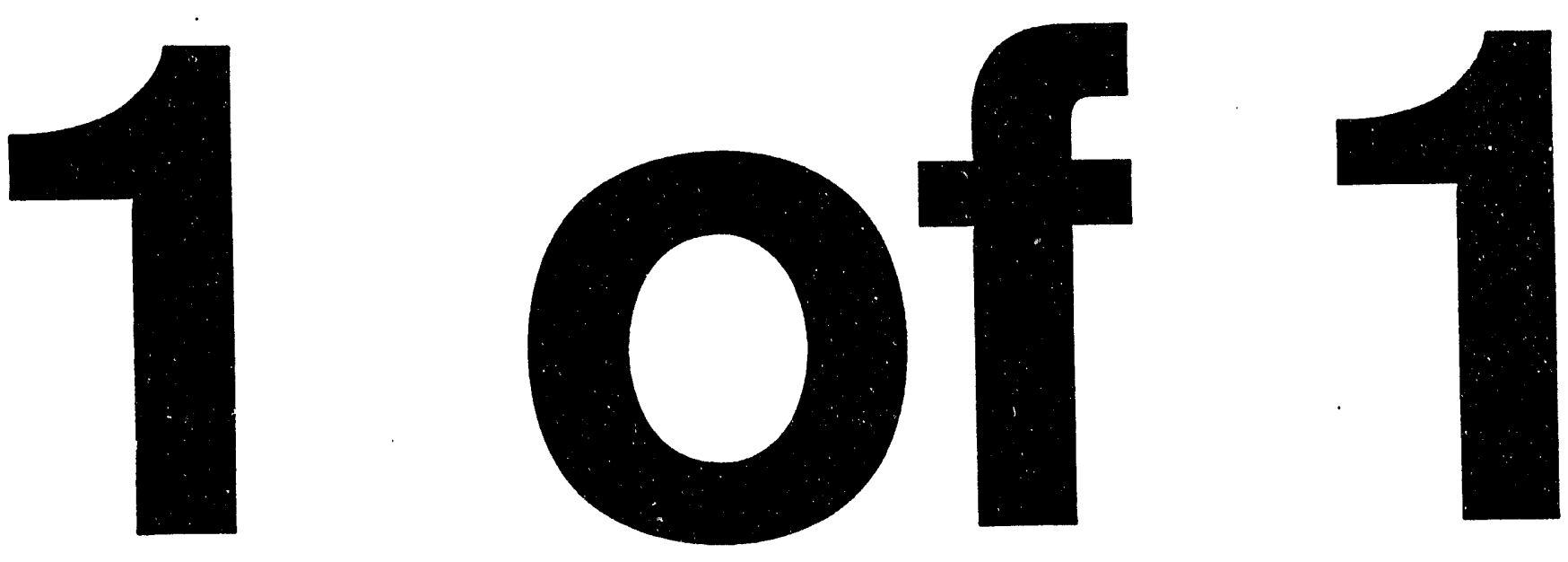
DOE/PC/91297- TS $^{-5}$

\author{
Quarterly Technical Progress Report
}

(December 1, 1993 to February 28, 1994)

\author{
A COMPUTATIONAL MODEL FOR \\ COAL TRANSPORT AND COMBUSTION \\ Grant Number: DE-FG22-91PC91297
}

\author{
Goodarz Ahmadi \\ Department of Mechanical and Aeronautical Engineering \\ Clarkson University
}

Submitted to

U.S. Department of Energy

Pittsburgh Energy Technology Center

\author{
Attention: \\ Dr. Mehrdad Massoudi \\ Project Officer \\ DISCLAIMER
}

$\checkmark$ This report was prepared as an account of work sponsored by an agency of the United States Government. Neither the United States Government nor any agency thereof, nor any of their employees, makes any warranty, express or implied, or assumes any legal liability or responsibility for the accuracy, completeness, or usefulness of any information, apparatus, product, or process disclosed, or represents that its use would not infringe privately owned rights. Reference herein to any specific commercial product, process, or service by trade name, trademark, manufacturer, or otherwise does not necessarily constitute or imply its endorsement, recommendation, or favoring by the United States Government or any agency thereof. The views 


\section{A COMPUTATIONAL MODEL FOR}

\section{COAL TRANSPORT AND COMBUSTION}

Grant Number: DE-FG22-91PC91297

Project Period: September 1, 1991 to August 31, 1994

Contract Recipient: Clarkson University

Project Principal Investigator: Goodarz Ahmadi

DOE Project Officer: Dr. Mehrdad Massoudi

\section{SUMMARY}

In the period of December 1, 1993 to February 28, 1994, considerable progress in the experimental study of mono-granular layer simple shear flow device was made. Experimental data concerning the mean granular velocity, fluctuation velocity and solid volume fraction were obtained. The resulting data revealed new interesting features of particulate flows.

The thermodynamically consistent, rate dependent model for turbulent two-phase flows and its application to analysis of simple shear flow was completed. Further progress on the application of the kinetic model for rapid flows of granular materials including the frictional energy losses were made. The flow over a vibrating plate was analyzed and the velocity, the fluctuation energy and the solid volume fraction profiles were evaluated.

A computational model for analyzing flow of granular materials in ducts and passages with bumpy walls was developed. The special cases of Couette and chute flows was analyzed. The results were compared with the experimental data and good agreement was observed.

Flows of gas-solid mixtures in vertical ducts were studied. A computational model for analyzing two-phase flow was developed, and the phasic mean velocity and fluctuation energy profiles were evaluated. The results were compared with the experimental data of Tsuji an co-worker and good agreement was obtained.

Rapid granular flows around a submerged rectangular block in a channel were described. It was shown that the model can predict the features of the flows in complex geometry regions. 


\section{PROGRESS REPORT}

\section{OBJECTIVES}

The objective of this project is to develop an accurate model describing turbulent flows of coal slurries, rapid flows of granular coal-air mixtures, and turbulent coal combustion processes. The other main objective is to develop a computer code incorporating the new model. Experimental verification of the foundation of the model is also included in the study.

\section{SIGNIFICANCE TO FOSSIL ENERGY PROGRAM}

A completely satisfactory theory describing the bulk coal transport including the interstitial fluid effects does not exist. This is particularly the case for turbulent flows of dense coal particle-liquid mixtures and chemically active coal combustor flows. Coal slurry and bulk transports, and operation of coal combustors accounts for a substantial portion of the cost of coal energy conversion systems. The major increase in cost arises from the need to over-design these facilities to guarantee reliability. Understanding the flow behavior of relatively dense coal slurries and bulk solids in various geometries including coal combustors, are indispensable to economical design of the needed equipment. This project aims to develop a sound practical model for coal transport and combustion. In addition, a computational predictive capability for analyzing rapid flows of granular coal particles, and reacting and nonreacting turbulent flows of dense or dilute multiphase coal mixtures will be provided. 


\section{HIGHLIGHT OF THE EARLIER ACCOMPLISHMENTS}

An experimental setup for generating simple shear flows of a mono-granular layer was constructed. Preliminary experimental data for mean velocity, fluctuation energy and solid volume fraction for shearing of $12 \mathrm{~mm}$ multi-color glass particles were obtained.

Thermodynamically admissible expressions for the phasic stress tensors, heat and fluctuation energy flux vectors for turbulent multiphase flows were derived. The material parameters of the model were evaluated from the limiting conditions of rapid flows of dry spherical granular particles, and single-phase turbulent fluid flow. The case of simple shear flows of glass beads-water mixtures was studied.

A thermodynamically consistent model for rapid flow of granular materials in a rotating frame of reference, along with a transport equation for the granular kinetic stress tensor were developed. The model parameters for the special case of spherical nearly elastic particles were evaluated. The results for the granular stresses and the normal stress differences were compared with the available simulation data and good agreement was observed.

Effects of frictional loss of energy on rapid granular shear flow's were studied. The previously developed kinetic based model was used and the mean velocity, the fluctuation kinetic energy and the solid volume fraction profiles were evaluated under a variety of conditions and different friction coefficients.

The computational model for rapid granular and two-phase flows in complex geometries was further developed. The discrete element scheme was used and the granular flow down a chute was analyzed. The results were compared with the experimental data model prediction of Savage, and the existing simulation results, and good agreements were observed. Progress has been made in developing an appropriate computational model for analyzing turbulent two-phase 
flows with various loadings.

\section{A THERMODYNAMICALLY CONSISTENT RATE-DEPENDENT MODEL FOR TURBULENT TWO-PHASE FLOWS}

A thermodynamically consistent theory for dense two-phase flow was developed. The new model includes the transport equations for the phasic fluctuation kinetic energies. Anisotropic and rate-dependent expressions for the phasic stress tensors were also derived. For dense solid-liquid mixtures, the material parameters of the model were evaluated from the limiting conditions. The special case of a simple shear flow of glass beads-water mixtures was studied. The coefficient of restitution for glass bead collision in water was experimentally measured and used in the model predictions. The details of the model equations were described in the earlier reports.

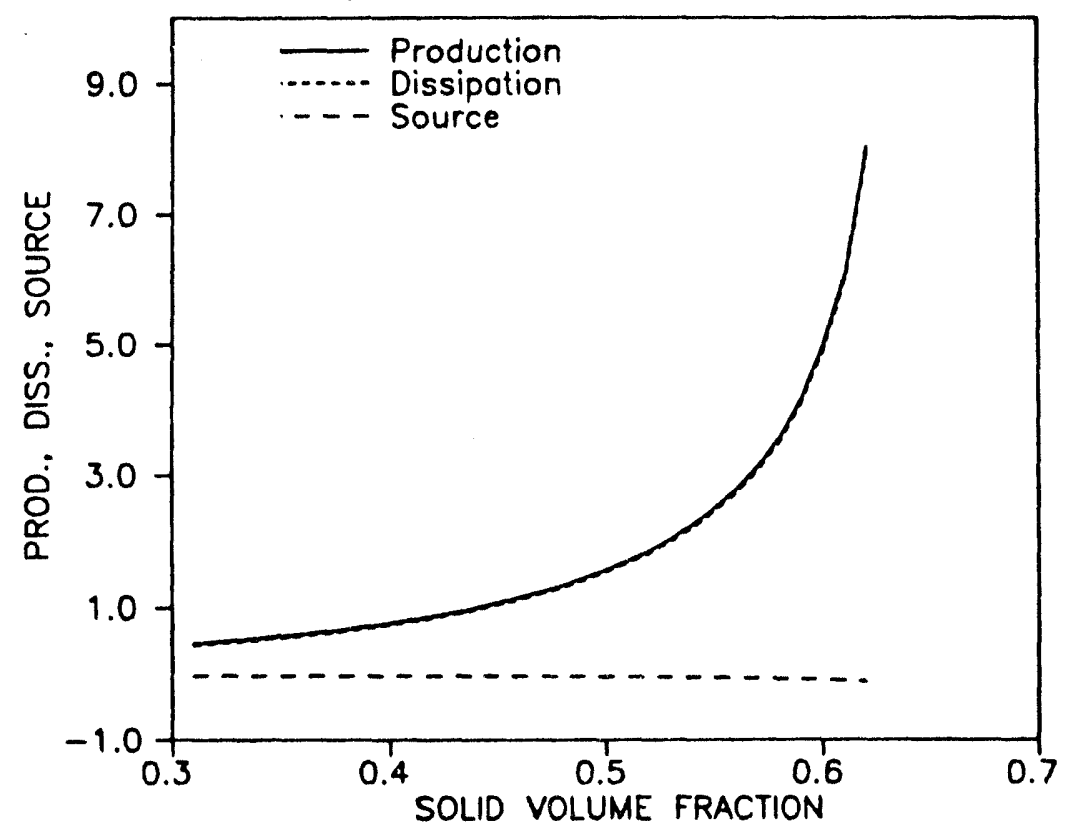

Figure 1. Variations of nondimensional fluctuation energy production, dissipation and source terms of the particulate phase with solid volume fraction for $1.1 \mathrm{~mm}$ glass beads in water. 
Figures 1 and 2 show variations of nondimensional phasic fluctuation energy production, dissipation and source terms with solid volume fraction for a mixture of $1.1 \mathrm{~mm}$ glass beads in water. Figure 1 shows that the nondimensional particulate fluctuation energy production and dissipation increases rapidly with an increase in solid volume fraction. The source term for the particulate phase is negative and increases with $v$. The fluid phasic fluctuation energy production and dissipation, however, decreases as $v$ increases. The fluctuation energy source term is positive for the fluid phase and increases slightly with solid volume fraction.

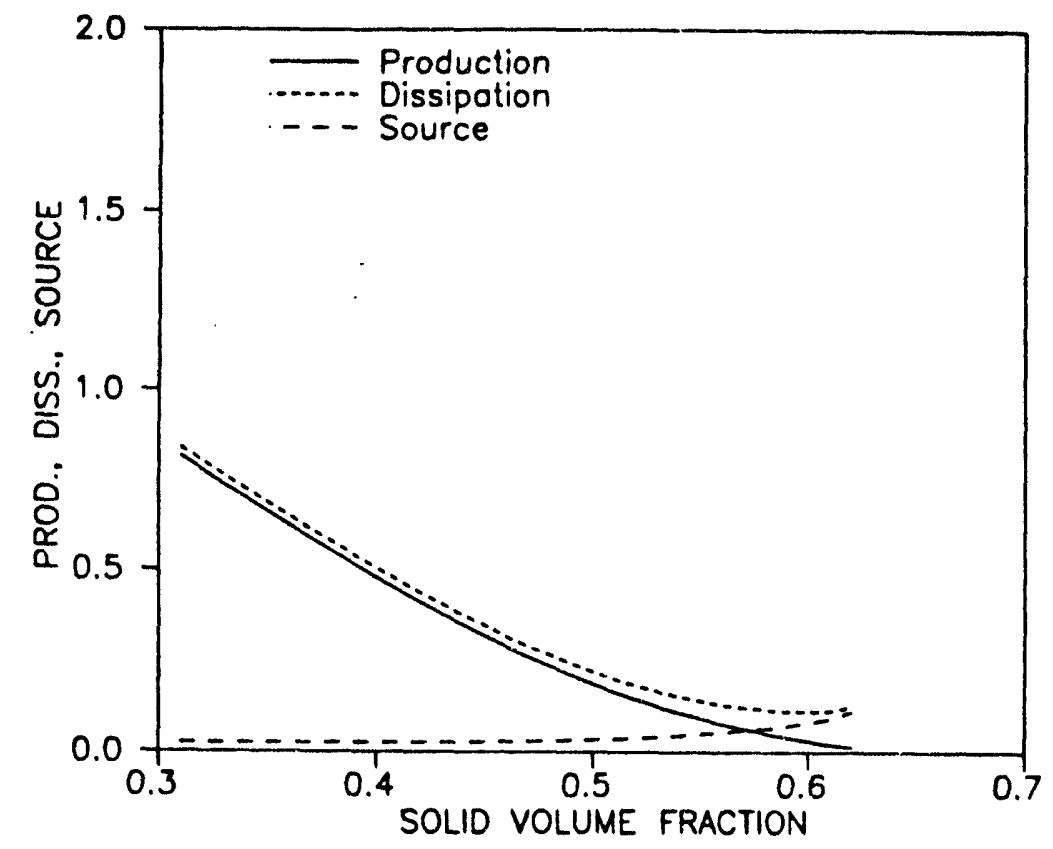

Figure 2. Variations of nondimensional fluctuation energy production, dissipation and source terms of the fluid phase with solid volume fraction for $1.1 \mathrm{~mm}$ glass beads in water.

For smaller values of $v$, the fluid phasic energy production and dissipation become rather large and that of the solid phase decreases. At large values of solid volume fraction (near the maximum packing conditions with $v$ close to 0.64 ), these figures show that the fluctuation energy is mainly produced and dissipated by the particulate phase and the contribution of the fluid phase 
is negligible.

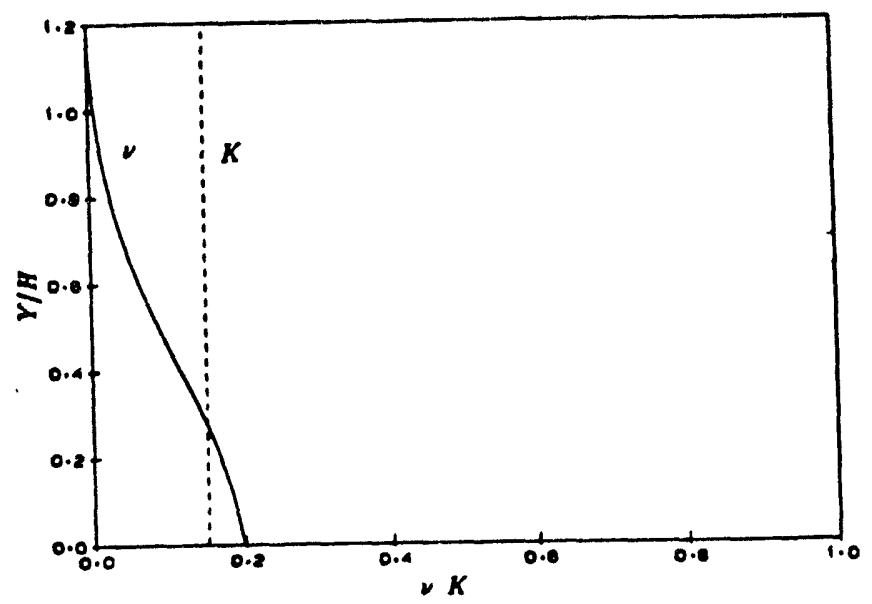

Figure 3. Variations of fluctuation energy, and solid volume fraction profiles for granular flows over a vibrating plate.

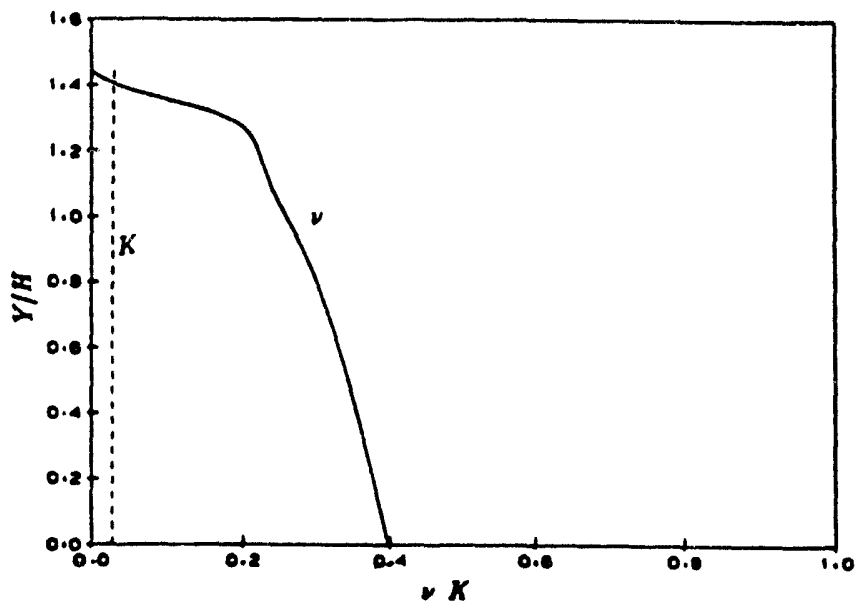

Figure 4. Variations of fluctuation energy, and solid volume fraction profiles for granular flows over a vibrating plate. 


\section{ANALYSIS OF RAPID GRANULAR FLOWS INCLUDING FRICTIONAL LOSSES}

The kinetic model for particulate flows, including frictional energy losses, was used to analyze granular flows over a vibrating plate. The equations of motion were solved using a fourth-order Runge-Kutta numerical scheme. The corresponding mean velocity and solid volume fraction profiles are shown in figures 3 and 4 . It is observed that the predicted fluctuation energy profile has a rather high value near the vibrating plate and decreases with distance form the surface. The solid volume fraction has a similar trend of variation. It has a rather high value near the plate, and decreases to zero near the free surface.

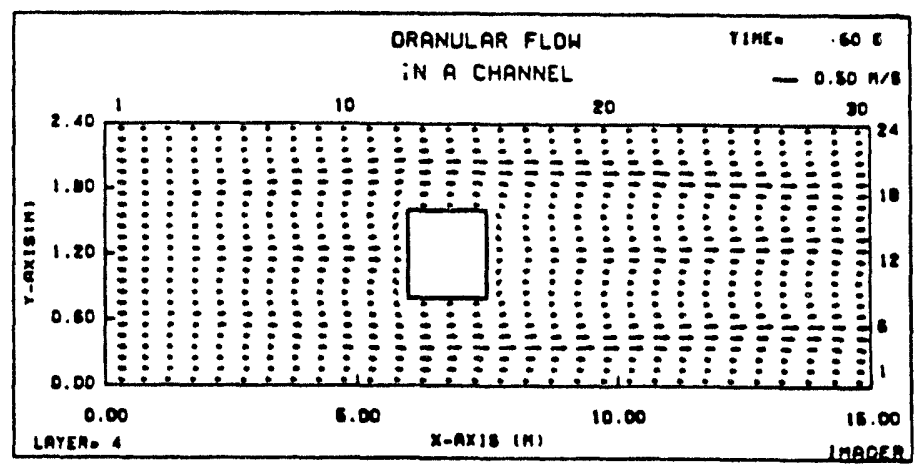

Figure 5. Velocity vector plot at horizontal $x y$-plane that crosses the surface of the block at $t=60 \mathrm{sec}$.

\section{COMPUTATIONAL MODEL DEVELOPMENT}

The goal of this phase of the study is to develop an appropriate computational scheme 
for solving granular and two-phase flows.

\section{Granular Flows in Complex Domain}

A computational model for analyzing granular flow in complex regions was developed.

The special case of granular flow in a chute with a rectangular block is studied. The details of the computational model and the discretization were described in the earlier report. The special case of granular flows in a channel with a submerged block is described in this section.

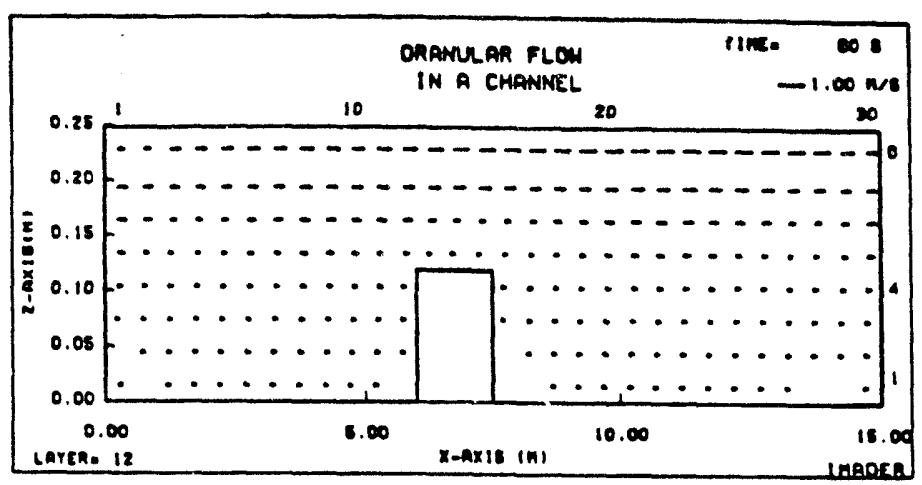

Figure 6. Velocity vector plot at vertical $x z$-plane at the channel centerline at $t=60 \mathrm{sec}$.

The simulation results for the velocity and solid volume fraction fraction field are shown in Figures 5-7. Figure 5 shows the velocity vector plot in the horizontal $x y$-plane that crosses the surface of the submerged block at $t=60 \mathrm{sec}$. The velocity field in the vertical $x z$-plane at the middle of the channel is shown in figure 7. It is observed that the block significantly distorts the 
flow field. In particular, on both sides of the block, the flow accelerates and behind the block a wake is formed. Figure 7 shows the solid volume fraction contour plot in the plane of symmetry of the channel at time $t=60 \mathrm{sec}$. It is observed that flow is stratified and the solid volume fraction increases with depth.

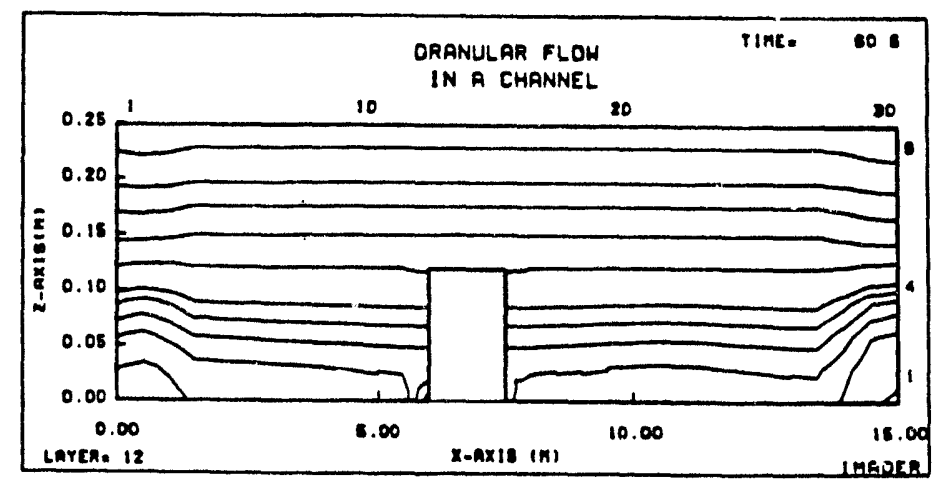

Figure 7. Solid volume fraction contours at $t=60 \mathrm{sec}$.

\section{Granular Flows with Bumpy Boundary}

Interaction of particles with the boundary is known to significantly affect flow and transport of granular materials. The kinetic model of granular materials including frictional losses was used in the analysis. The presence of bumpy boundary conditions leads to a strongly couple system of governing equations which has to be solve numerically even for simple case of Couette flow. As noted in the earlier report, a special discretization scheme for evaluating the granular flow field was developed. The computational model was used and the mean velocity, the 
fluctuation kinetic energy and the solid volume fraction profiles for granular flows between two parallel walls were evaluated. The results for different values of friction coefficients are plotted in figure 8. The molecular dynamics (MD) simulations of Savage and Dai (1992) which are reproduced in figure 8 show reasonable agreement with the model predictions for $\mu=0$.
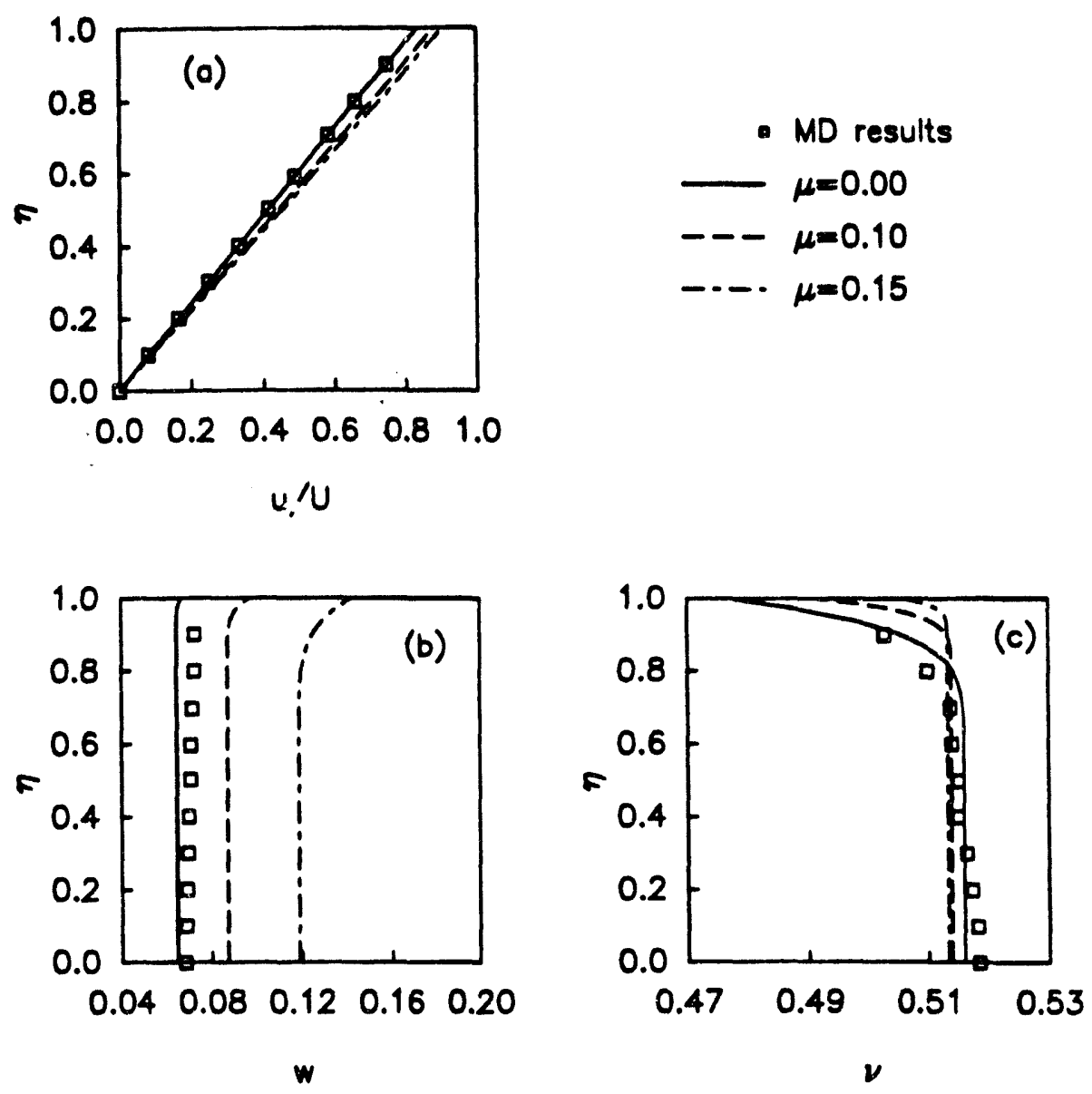

Figure 8. Variations of mean velocity, fluctuation energy and solid volume fraction profiles. Comparison with the molecular dynamic simulation of Savage and Dai (1992).

Figure 8a also indicates that the mean velocity profile for nearly elastic particles is roughly linear. Comparison with the results reported in the earlier report shows that the slip 
velocity is reduced as restitution coefficient; $r$, increases. The SR-fluctuation kinetic energy also increases rapidly as $r$ increases. The effect of variation of friction coefficient, $\mu$, is quite small for velocity and concentration profiles. However, figure $8 \mathrm{~b}$ shows that a small increase in $\mu$ leads to a significantly higher fluctuation kinetic energy field.

As noted before, figure $8 \mathrm{a}$ also show that there are a significant amount of slip at the wall. The slip velocity decreases as the friction coefficient increases. Figure $8 \mathrm{~b}$ indicates that the fluctuation velocity has a gradient near the wall and approaches a constant away from the wall. The solid volume fraction, is rather small near the wall and increases with distance from the wall. Figure $8 \mathrm{c}$ shows that $\nu$ is roughly constant in the core region. 'These results show that the commonly used assumption that the kinetic energy and the solid volume fraction are constants for Couette flows of granular materials is not quite correct.

\section{Granular Gravity Flows}

Granular gravity flows over an inclined bumpy chute is analyzed. The earlier developed kinetic-based model for rapid flows of granular materials which includes the frictional losses of energy during particle-particle and/or particle-wall collisions is used in the analysis. The predicted mean velocity, fluctuation energy, and solid volume fraction profiles are evaluated and the results are compared with existing experimental data.

Figure 9 compares the experimental data of Drake and Shreve (1986) with the predicted mean velocity, solid volume fraction and SR-fluctuation kinetic energy profiles for a chute inclination angle of $\alpha=42.75^{\circ}$. The experiment was performed in an inclined glass-walled channel $3.7 \mathrm{~m}$ long and $6.5 \mathrm{~mm}$ wide using $6 \mathrm{~mm}$ diameter cellulose-acetate spherical particles. The bed consisted of spheres like those in the flows, which were glued with random spacing to an aluminum bar. A granular layer height to particle diameter ratio of $H / d=16$, a particle-particle 
restitution coefficient $r=0.9$, particle-wall restitution coefficient $r_{w}=0.5$ and $\mu u=\mu u_{w}=0.3$ are used in the numerical simulation. It is observed that the predicted velocity and solid volume fraction profiles are in good agreement with the experimental data. The predicted SR-fluctuation kinetic energy profile, however, overestimates the experimental data near the wall. As noted before, the experiment was performed for a mono-layer of granular particles in a two-dimensional channel, while the present model predictions are for fully three-dimensional particulate flows. Therefore, certain deviations should be expected.

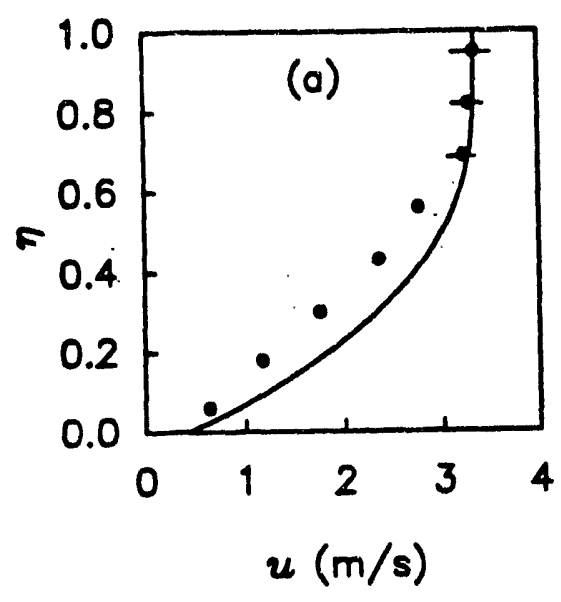

$H / d=16$

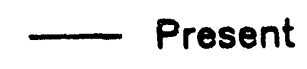

- Drake \& Shreve
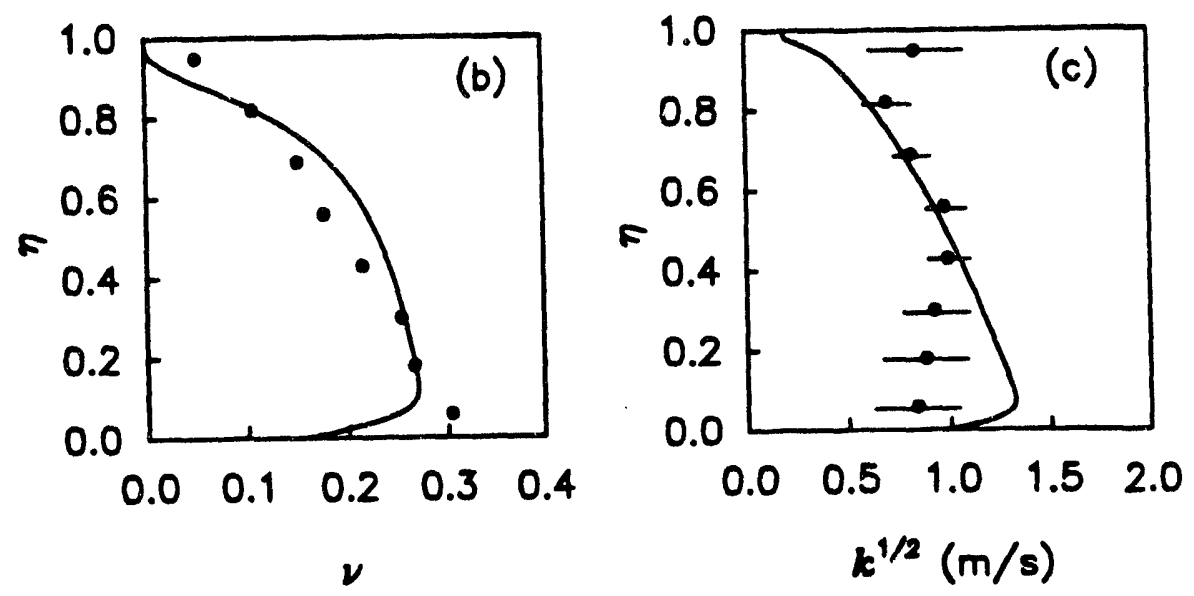

Figure 9. Sample prediction results for granular over a bumpy incline. Comparison with the experimental data of Drake and Shreve (1986). 


\section{TWO-PHASE FLOWS}

In this section, model predictions for mean gas velocity, mean particle velocity, and phasic turbulence intensities are presented and compared with the experimental results of Tsuji et al. (1984). Using a Laser-Doppler Velocimeter (LDV), Tsuji et al. reported measurements of the phasic flow properties in a fully developed, two-phase, air-particle turbulent flow in a $30.5 \mathrm{~mm}$ vertical pipe. In their experiments, polystyrene spheres with density of $\rho_{0}=1020 \mathrm{~kg} / \mathrm{m}^{3}$ and diameters in the range $0.2 \mathrm{~mm}$ to $1 \mathrm{~mm}$ were suspended in air.

A restitution coefficient of $r=0.9$, for particle-particle collisions, and $r_{w}=0.7$, for particle-wall collisions and a coefficient of dynamic friction $\mu=0.2$ between a particle and the wall are used in the present study. These values are consistent with the observation of Govan et al. (1989), who studied the trajectories of glass spheres transported in a small pipe under conditions similar to those of Tsuji et al. (1984).

Figure 10 presents the model predictions for the phasic flow conditions of air and $0.2 \mathrm{~mm}$ polystyrene sphere mixtures. Here, the mass loading ratio is one, the centerline gas velocity is 18.9 $\mathrm{m} / \mathrm{s}$, and the flow Reynolds number based on mean ga.; velocity is 30,000 . At this low solid volume fraction, figure $10 \mathrm{a}$ indicates that the gas velocity profile resembles that of a turbulent flow with a sharp gradient near the wall. The mean particle velocity profile is quite flat across the duct, with a significant slip velocity at the wall. In most parts of the channel, the particle velocity is smaller than the air velocity, except near the wall, where it becomes larger. This is because the air flow is subject to the no-slip condition at the wall, while the particle phase slips. The air and particle mean velocity profiles are compared with the experimental data of Tsuji et al. (1984) in figure 10a. While the predictions are in good agreement with the data, the model slightly over-predicts the gas velocity and under-predicts the particle velocity. 


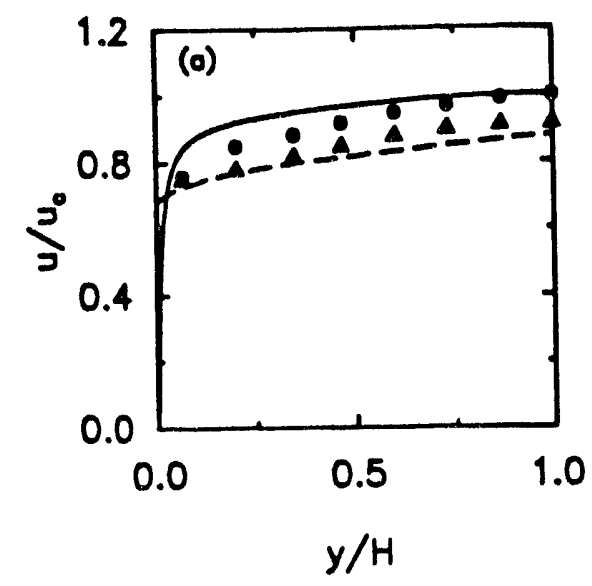

$$
\begin{array}{r}
\text { Re }=3.0 \times 10^{4}, \delta=0.2 \mathrm{~mm} \\
m=1.0, u_{c}=18.9 \mathrm{~m} / \mathrm{s} \\
-- \text { Prediction (porticle) } \\
\text { - prediction (oir) } \\
\text { - Tsuji et al. (porticle) } \\
\text { - Tsuji et al. (oir) }
\end{array}
$$
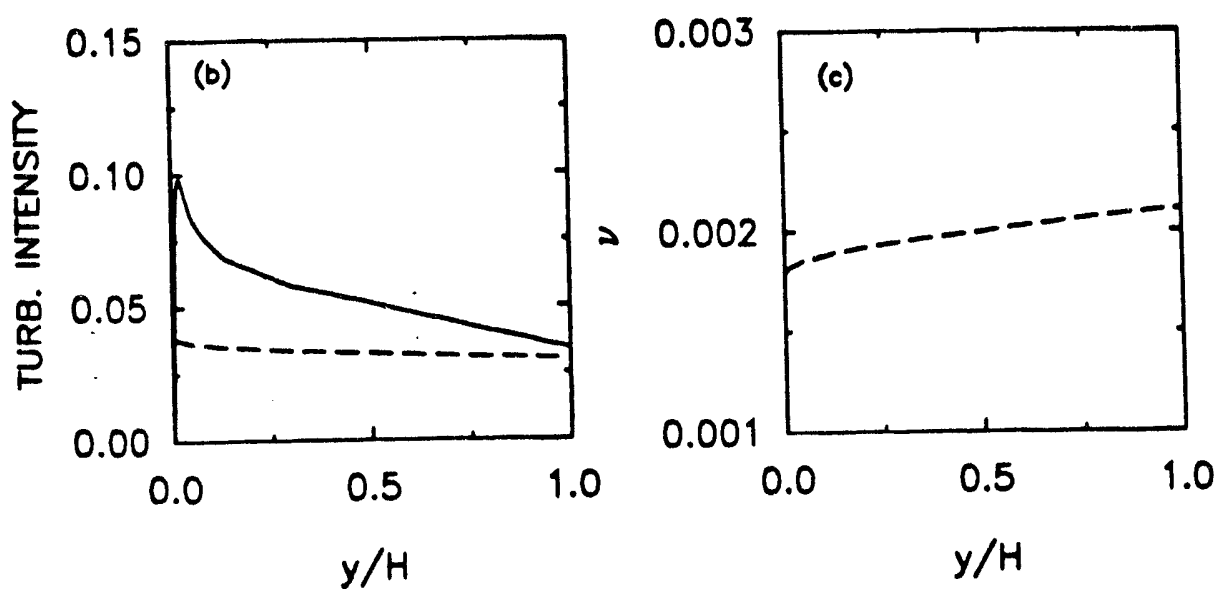

Figure 10. Sample prediction results for two-phase flows in a duct. Comparison with the experimental data of Tsuji et al. (1984).

Figure $10 \mathrm{~b}$ shows the computed turbulence intensities of air and particulate phases. It is observed that the turbulence intensity of particulate phase is smaller than that of the air, and has a flat profile across the channel. The gas turbulence intensity profile has the shape similar to that of a clear gas. Additional results (not shown here) indicated that the relative air turbulence intensity in the duct core is decreased. Thus, in this case, the presence of particles suppresses the air turbulence intensity. Figure $10 \mathrm{c}$ shows the solid volume fraction profile across the channel. It is observed that the solid volume fraction is quite low (of the order of 0.002 ) and the particle 
concentration gradually increases toward the center of the channel. Unfortunately, the experimental data for turbulence intensity and concentration profiles were not reported for comparison.

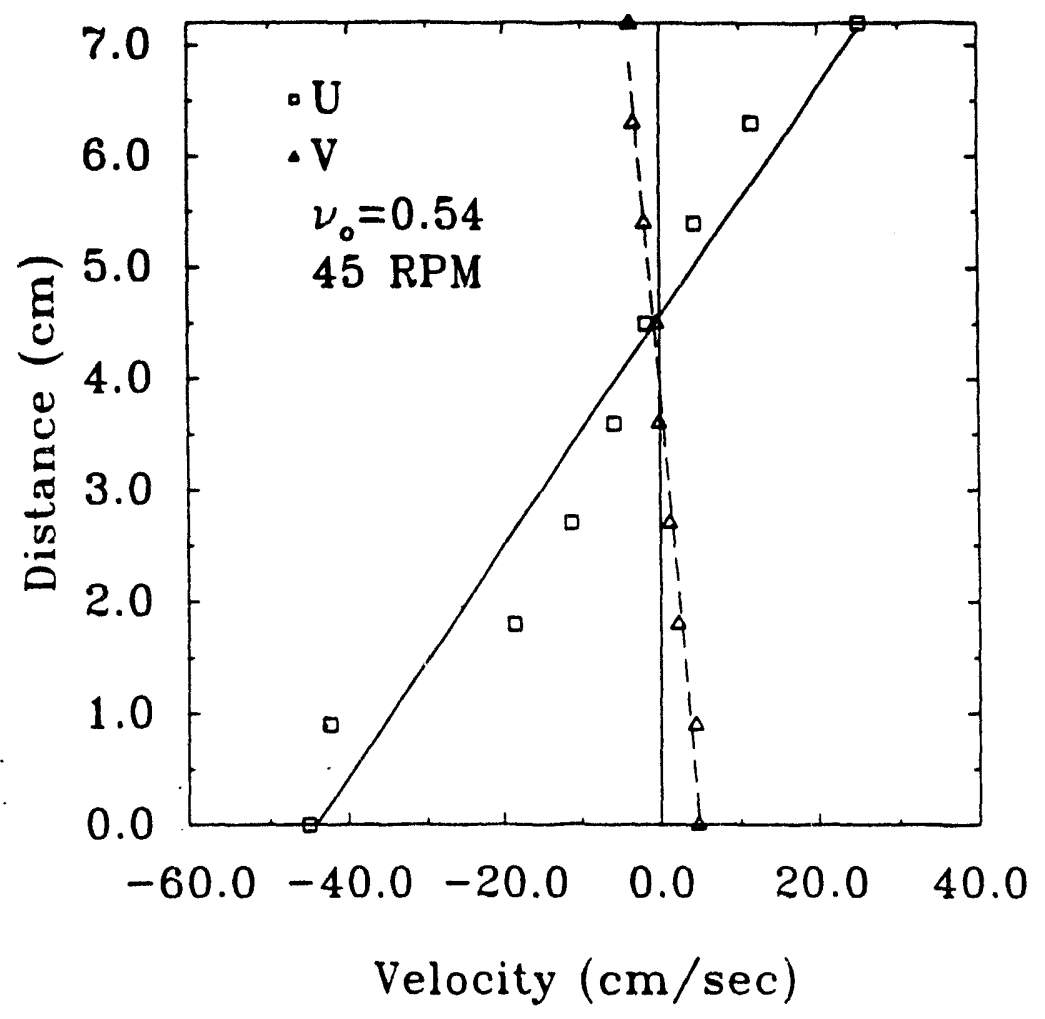

Figure 11. Experimental data for the mean velocity profile.

\section{EXPERIMENTAL STUDY}

A mono-granular simple shear flow setup was constructed and was used for the experimental study. A collection of multi-colored spherical glass balls which are $12 \mathrm{~mm}$ in diameter were used as granular particles. A video camera is used to record the motions of particles. For different shear rates, the position of the balls in consecutive frames taken $1 / 30$ th of a second apart were measured. Using this technique, the velocity vector of each particle was calculated. The shearing region was divided into 10-15 equivalent horizontal segments. Averaging procedures are used to provide the experimental velocity and concentration profiles. 


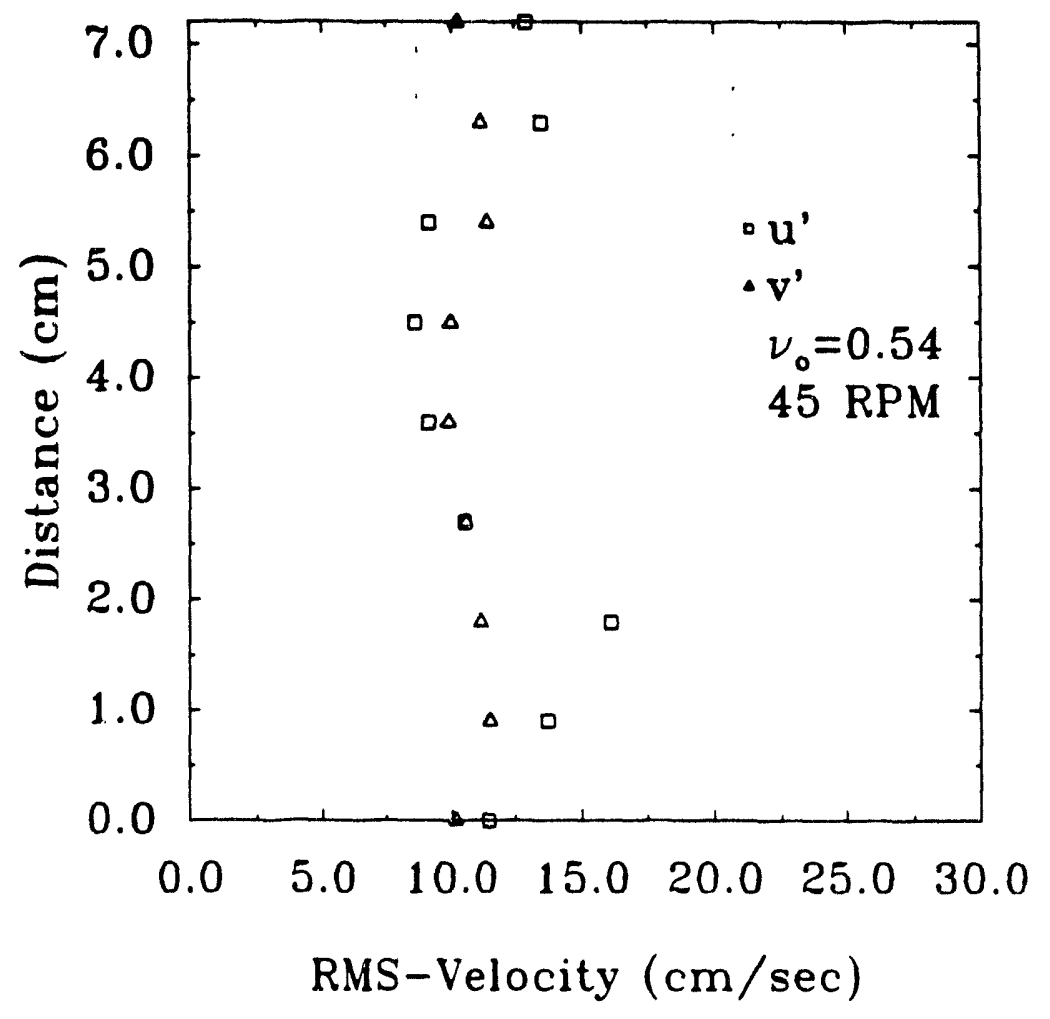

Figure 12. Experimental data for the RMS-fluctuation velocities.

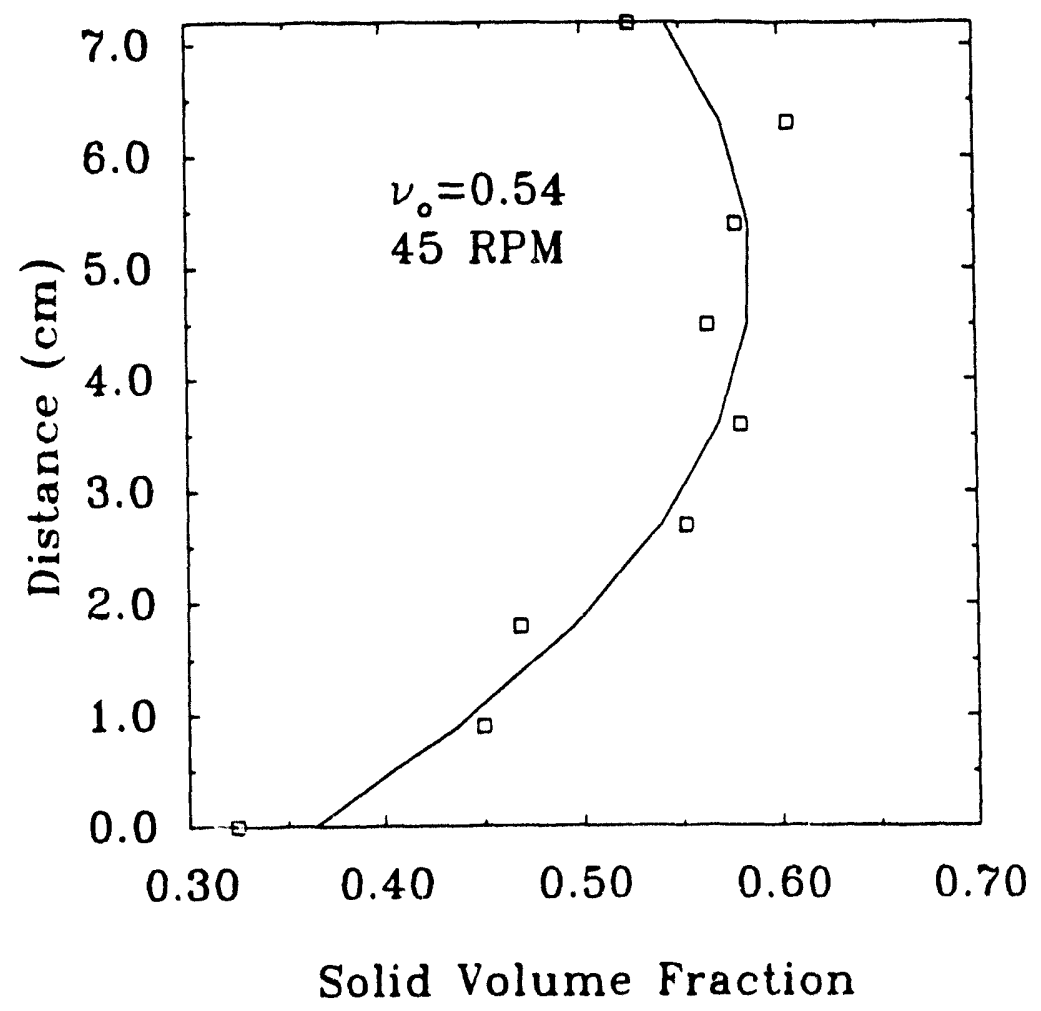

Figure 13. Experimental data for the solid volume fraction profile. 
Figures 11-13 show sample experimental data for mean velocity, concentration and fluctuation velocity profiles. It is observed that the significant amount of slip exist. The slip is much larger at the inner wall when compared with that of the outer wall. Figure 11 also shows that the axial velocity is roughly linear in the central part of the shearing region. From figures 12 and 13 It is observed that the solid volume fraction and fluctuation energy varies across the section.

\section{BIBLIOGRAPHY OF PUBLICATIONS EMANATING FROM THIS PROJECT}

\section{Journals Publications}

G. Ahmadi and S.J. Chowdhury, "A Rate-Dependent Algebraic Stress Model for Turbulence," Applied Math. Modelling 15, 516-524 (1991).

H. Ounis and G. Ahmadi, "Motions of Small Particles in a Turbulent Simple Shear Flow Field Under Microgravity Condition," Physics of Fluids A $\underline{3}, 2559-2570$ (1991).

S. Abu-Zaid and G. Ahmadi, "A Thermodynamically Consistent Stress Transport Model for Rotating Turbulent Flows," Geophys. Astrophys. Fluid Dynamics 61, 109-121 (1991).

G. Ahmadi, "A Thermodynamically Consistent Rate-Dependent Model for Turbulence, Part I Formulation," Int. J. Non-Linear Mech. 26, 595-607 (1991).

K.A. Elrais, W. Eckerle, G. Ahmadi and A.H. Eraslan, "Simulation of Transient Three-Dimensional Natural Convection and Saturated Pool Boiling, Int. J. Numerical Methods Heat Fluid Flow 2, $139-154$ (1992).

S.J. Chowdhury and G. Ahmadi, "A Thermodynamically Consistent Rate-Dependent Model for Turbulence, Part II - Numerical Results," Int. J. Non-Linear Mech. 27, 705-718 (1992). 
W.G. Paff and G. Ahmadi, "On Convergence of Karhunen-Loeve Series Expansion for a Brownian Particle," J. Appl. Mech. Trans. ASME (In Press).

S. Abu-Zaid and G. Ahmadi, "A Stress Transport Model for Granular Flows in a Rotating Frame, Int. J. Engng. Sci. 30, 1483-1495 (1992).

S.J. Chowdhury and G. Ahmadi, "Analysis of A Mixing Layer by a Rate-Dependent Turbulence Model," ASCE, Journal of Engineering Mechanics 119, 1700-1706 (1993).

D. Ma, A.H. Eraslan and G. Ahmadi, "A Computer Code for Analyzing Transient Three-Dimensional Rapid Granular Flows in Complex Geometries, Computer Fluids 22, 25-50 (1993).

S. Abu-Zaid and G. Ahmadi, "Analysis of Rapid Shear Flows of Granular Materials by a Kinetic Model Including Frictional Losses," Powder Technology (In Press).

S. Abu-Zaid and G. Ahmadi, "A Thermodynamically Consistent Rate-Dependent Model for Turbulent Two-Phase Flows, Part I - Formulation," MAE Report-281 November (1993). Also submitted to Int. J. Multiphase Flows.

S. Abu-Zaid and G. Ahmadi, "A Thermodynamically Consistent Rate-Dependent Model for Turbulent Two-Phase Flows, Part II - Simple Shear Flows," MAE Report-282 November (1993). Also submitted to Int. J. Multiphase Flows.

\section{Conference Presentations}

G. Ahmadi and S. Abu-Zaid, "Overview of Thermodynamical Approach to Modeling Turbulent Flows of Two-Phase Solid-Liquid Mixtures," International Conference on Multiphase Flow '91-Tsukuba, Tsukuba, Japan, September 24-27, 1991. 
G. Ahmadi and S. Abu-Zaid, "A Thermodynamically Consistent Model for Turbulent Two-Phase Flows," 28th Annual Technical Meeting of the Society of Engineering Science, Gainsville, FL, November 6-8, 1991.

G. Ahmadi and S. Abu-Zaid, "Analysis of Rapid Shear Flows of Granular Materials by a Stress Transport Model," 28th K.nnual Technical Meeting of the Society of Engineering Science, Gainsville, FL, November 6-8, 1991.

G. Ahmadi, "Overview of Thermodynamical Modeling of Turbulent Flows of Multiphase Mixtures," Proceedings of the International Conference on Engineering Application of Mechanics, Vol. 3, Sharif University of Technology, Ed. by M.S. Sadeghipour et al., June 9-12, 1992, 386-399.

G. Ahmadi and S. Abu-Zaid, "A Thermodynamically Consistent Stress Transport Model for Rapid Granular Flows," The 23nd Annual Meeting of the Fine Particle Society, Las Vegas, Neyada, July 13-17, 1992.

G. Ahmadi and S. Abu-Zaid, "A Model for Turbulent Two-Phase Flows," MEET'N'93, ASME/ASCE/SES Joint Meeting, University of Virginia, Charlottsville, VA, June 6-9, 1993.

G. Ahmadi, D. Ma, and A. Eraslan "Rapid Granular Flow in Complex Geometry Regions," MEET'N'93, ASME/ASCE/SES Juint Meeting, University of Virginia, Charlottsville, VA, June 6-9, 1993.

G. Ahmadi, "A Computational Model for Coal Transport and Combustion," University Coal Research Contractors" Review Conference, Pittsburgh, PA, June 23-25, 1993.

G. Ahmadi and S. Abu-Zaid, "Turbulent Two-Phase Flow of Dense Mixtures-A Thermodynamically Consistent Model," The 24th Annual Meeting of the Fine Particle Society and First International Conference on Pharmaceutical Sciences and Technology, Chicago, Illinois, August 23-28, 1993. 
J. Cao, and G. Ahmadi, "Numerical Simulation of Granular Couette Flows Between Two Rough Parallel Plates," The 24th Annual Meeting of the Fine Particle Society and First International Conference on Pharmaceutical Sciences and Technology, Chicago, Illinois, August 23-28, 1993.

G. Ahmadi, D. Ma, and A. Eraslan "Analysis of Transient Rapid Granular Flows in a Channel with an Obstruction," The 24th Annual Meeting of the Fine Particle Society and First International Conference on Pharmaceutical Sciences and Technology, Chicago, Illinois, August 23-28, 1993. 

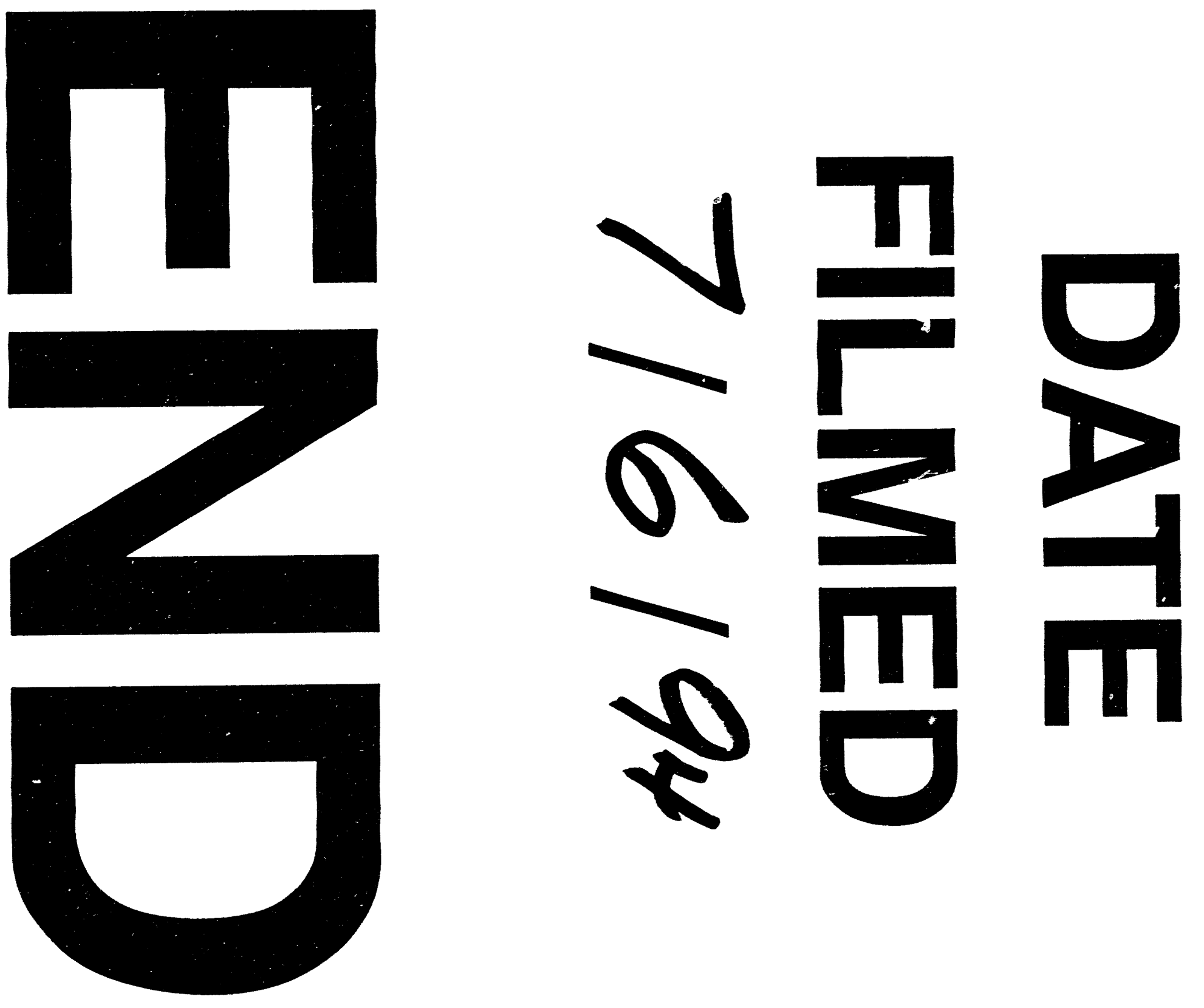

to 
